\title{
Highly sensitive silicon microreactor for catalyst testing
}

Henriksen, Toke Riishøj; OIsen, Jakob Lind; Vesborg, Peter Christian Kjærgaard; Chorkendorff, Ib; Hansen, Ole

Published in:

Review of Scientific Instruments

Link to article, DOI:

$10.1063 / 1.3270191$

Publication date:

2009

Document Version

Publisher's PDF, also known as Version of record

Link back to DTU Orbit

Citation (APA):

Henriksen, T. R., Olsen, J. L., Vesborg, P. C. K., Chorkendorff, I., \& Hansen, O. (2009). Highly sensitive silicon microreactor for catalyst testing. Review of Scientific Instruments, 80(12), 124101.

https://doi.org/10.1063/1.3270191

\section{General rights}

Copyright and moral rights for the publications made accessible in the public portal are retained by the authors and/or other copyright owners and it is a condition of accessing publications that users recognise and abide by the legal requirements associated with these rights.

- Users may download and print one copy of any publication from the public portal for the purpose of private study or research.

- You may not further distribute the material or use it for any profit-making activity or commercial gain

- You may freely distribute the URL identifying the publication in the public portal

If you believe that this document breaches copyright please contact us providing details, and we will remove access to the work immediately and investigate your claim 


\title{
Highly sensitive silicon microreactor for catalyst testing
}

\author{
Toke R. Henriksen, ${ }^{1,2}$ Jakob L. Olsen, ${ }^{2}$ Peter Vesborg, ${ }^{2}$ Ib Chorkendorff, ${ }^{2}$ and \\ Ole Hansen ${ }^{1,2, a)}$ \\ ${ }^{1}$ Department of Micro- and Nanotechnology, Technical University of Denmark, DTU Nanotech Building 345 \\ East, DK-2800 Kgs. Lyngby, Denmark \\ ${ }^{2}$ Department of Physics, Danish National Research Foundation's Center for Individual Nanoparticle \\ Functionality (CINF), Technical University of Denmark, Building 312, DK-2800 Kgs. Lyngby, Denmark
}

(Received 14 July 2009; accepted 6 November 2009; published online 7 December 2009)

\begin{abstract}
A novel microfabricated chemical reactor for highly sensitive measurements of catalytic activity and surface kinetics is presented. The reactor is fabricated in a silicon chip and is intended for gas-phase reactions at pressures ranging from 0.1 to 5.0 bar. A high sensitivity is obtained by directing the entire gas flow through the catalyst bed to a mass spectrometer, thus ensuring that nearly all reaction products are present in the analyzed gas flow. Although the device can be employed for testing a wide range of catalysts, the primary aim of the design is to allow characterization of model catalysts which can only be obtained in small quantities. Such measurements are of significant fundamental interest but are challenging because of the low surface areas involved. The relationship between the reaction zone gas flow and the pressure in the reaction zone is investigated experimentally. A corresponding theoretical model is presented, and the gas flow through an on-chip flow-limiting capillary is predicted to be in the intermediate regime. The experimental data for the gas flow are found to be in good agreement with the theoretical model. At typical experimental conditions, the total gas flow through the reaction zone is around $3 \times 10^{14}$ molecules s ${ }^{-1}$, corresponding to a gas residence time in the reaction zone of about $11 \mathrm{~s}$. To demonstrate the operation of the microreactor, $\mathrm{CO}$ oxidation on low-area platinum thin film circles is employed as a test reaction. Using temperature ramping, it is found that platinum catalysts with areas as small as $15 \mu \mathrm{m}^{2}$ are conveniently characterized with the device. () 2009 American Institute of Physics.
\end{abstract}

[doi:10.1063/1.3270191]

\section{INTRODUCTION}

In heterogeneous catalysis, the discovery, characterization, and optimization of catalysts require extensive experimentation and are critical steps in the development of new chemical processes. Traditionally, macroscale chemical reactors such as the idealized plug flow reactor have been used for catalyst testing. ${ }^{1}$ As a supplement to these traditional reactors, microfabricated reactors have, in recent years, shown promise as versatile analytical tools for measuring catalytic activity. Microfabricated reactors, also known as microreactors, are chemical reactors with dimensions in the micrometer range. Pioneered by, among others, Jensen et al., microreactors have been shown to offer a number of advantages compared to conventional macroscale reactors. ${ }^{2-6}$ The temperature of a microreactor can be varied easily and quickly because of the small size. Due to the high surface-to-volume ratio, heat transfer to and from the reaction zone is greatly enhanced. This reduces thermal gradients in the reactor and makes accurate control of temperature possible, even for strongly exothermic reactions. Furthermore, the small dimensions of the reactor improve mass transfer and reduce concentration gradients. Small thermal and concentration gradients are favorable when determining kinetic data. Reaction parameters such as pressure, residence time, and flow

${ }^{a)}$ Electronic mail: ole.hansen@nanotech.dtu.dk. rate are more easily controlled in reactions that take place in small volumes. In addition to these advantages, the small scale of the reactor improves safety of use for explosive mixtures and greatly reduces reactant gas consumption. Finally, it is possible to integrate heaters and sensors directly into microreactors using microfabrication technology.

Fundamentally, a microreactor consists of a system of microchannels in which the reactants will flow and react. The flow through the microchannels is mostly laminar, resulting in predictable and well-controlled flow patterns. Due to the small dimensions, the residence time of the reactant gas in the catalyst bed is typically low. Consequently, high space velocities can be obtained.

In combination with an appropriate instrument for gas composition analysis, it is possible to design microreactors which facilitate detection of very small amounts of gas phase products. This is a consequence of the low flow rate through the microchannel system. High sensitivity product detection under atmospheric pressure is useful in several respects. When investigating new, nonoptimized catalysts, the catalytic activity might be low, necessitating detection of small amounts of substances. Furthermore, some model catalysts, such as mass selected clusters and catalysts fabricated using electron-beam lithography (EBL), can only be obtained in small quantities. Thus, a sensitive experimental arrangement is required for measuring the catalytic activity of such samples. $^{7-9}$ 
A few microreactors for low-area catalyst characterization with gas-phase reactions have been reported in literature. Johansson et al. ${ }^{9}$ demonstrated a Pyrex flow microreactor for measuring catalytic activity of nanofabricated model catalysts at atmospheric pressure. The microreactor had a reaction chamber volume of around $100 \mathrm{~mm}^{3}$, a reaction zone gas flow of about $4 \times 10^{16}$ molecules s$~^{-1}$, and a gas residence time in the reaction zone of approximately $60 \mathrm{~s}$. They observed $\mathrm{CO}$ oxidation on a platinum wire with a surface area of $0.3 \mathrm{~mm}^{2}$. Jacobs et al. ${ }^{10}$ measured ethylene hydrogenation at atmospheric pressure in a closed tank reactor. They detected catalytic activity on an EBL-nanofabricated platinum model catalyst with a total active surface area of $4 \mathrm{~mm}^{2}$.

Our research group has previously fabricated microreactors and applied them in experiments concerning catalysis and surface science. ${ }^{11-14}$ In this paper, we present a novel continuous-flow microreactor with a highly improved sensitivity for measurements of catalytic activity. The device is intended for gas-phase reactions at pressures in the range of $0.1-5.0$ bar. The gas composition is analyzed using a quadropole mass spectrometer (QMS). As a characteristic feature, the microreactor presented here directs the entire reaction zone gas flow to enter the mass spectrometer. This increases the sensitivity considerably compared to traditional microreactors where only a small fraction of the gas flow is analyzed.

The microreactor is designed as an analytical tool for catalyst testing. In this context, the term catalyst testing covers measurements of catalytic activity, turnover frequency, apparent activation energy, and surface area, as a result of temperature programmed desorption and experiments with conversion rate as a function of temperature and gas phase stoichiometry. The high sensitivity of the reactor makes it especially well-suited for fundamental catalytic activity studies of model catalysts with low surface areas. A particularly important application is the characterization of mass selected metal clusters with a narrow size distribution. Such measurements are of significant interest for the purpose of correlating size and catalytic activity of clusters. ${ }^{15-17}$ Often, a low density of clusters is desirable in these studies to suppress sintering effects. Ensembles of metal clusters can be produced with a magnetron sputtering cluster source, but only in small quantities per unit time, since the cluster beam intensity decreases with reduced spread in the cluster size. Thus, a highly sensitive measurement technique, like the one presented here, could substantially reduce the time consumption of such experiments.

Although the high sensitivity is particularly advantageous for fundamental studies involving small quantities of model catalysts, the microreactor is not restricted to such experiments. In addition to deposition from a cluster source, catalyst samples can be introduced into the reactor using a variety of other methods, including flame spray deposition, ${ }^{18}$ dip-coating, spin coating, or manual deposition with a pipette. During catalyst deposition, a shadow mask can be used to confine deposition to the reaction chamber area. Any catalyst, which can be deposited with these methods and which can fit in the reaction chamber, can be tested with the device.

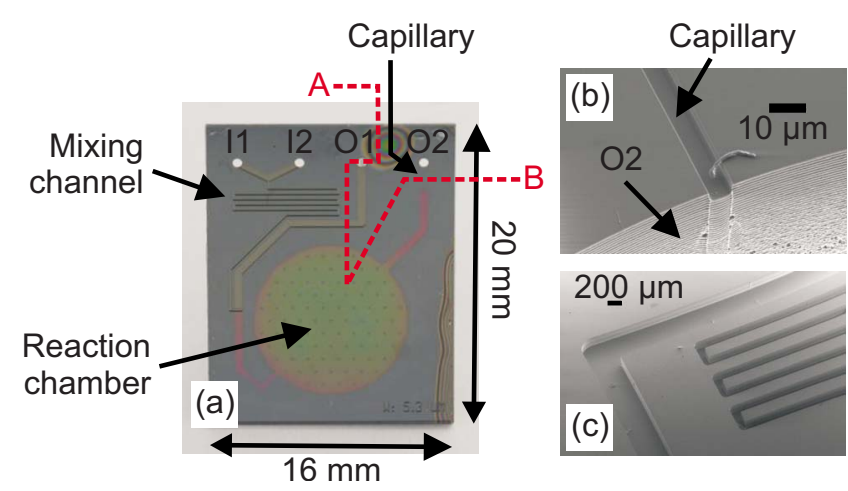

FIG. 1. (Color online) (a) Photograph of reactor showing the two inlets I1 and $\mathrm{I} 2$ and the two outlets $\mathrm{O} 1$ and $\mathrm{O} 2$. The dashed red line signifies the cross section shown in the process flow in Fig. 2. (b) Scanning electron micrograph of the junction between the capillary and the outlet O2. (c) Scanning electron micrograph of the mixing channel.

For example, many industrial catalysts with promoters, metal alloys, oxides, and supports can be conveniently tested with the microreactor.

In the work presented here, $\mathrm{CO}$ oxidation on circular platinum thin films of different areas is employed as a test reaction. The aim of these experiments is to demonstrate the operation of the reactor and the ability to characterize lowarea catalysts. While platinum thin films are not used as $\mathrm{CO}$ oxidation catalysts for practical purposes, they are often used as model systems and for test reactions in fundamental catalysis studies. ${ }^{19,20}$ Platinum thin films produced using electron-beam (e-beam) physical vapor deposition (PVD) and a lift-off process in acetone are used in this work because this is a convenient way of preparing catalysts with well controlled surface areas in the range 10-10000 $\mu^{2}$. The relation between the area of the catalyst circle and the lowest temperature at which catalytic activity can be detected in the QMS is investigated with reference to determining the sensitivity of the microreactor.

\section{EXPERIMENTAL}

\section{A. Microreactor design}

The microreactor directs the entire gas flow through the catalyst bed to a QMS, ensuring that almost all reaction products are present in the analyzed gas flow. Thus, unnecessary dilution of products is avoided, resulting in a high sensitivity. This concept places the following demands on the microreactor design: (1) the flow rate through the catalyst bed must be small, since gas can only be allowed to enter the QMS at a low rate. (2) Because of the low gas flow rate, the reaction zone volume must be small to ensure a relatively short gas residence time.

The microreactor consists of a microchannel system which has been formed in a silicon chip using reactive ion etching (RIE) and deep RIE (DRIE). DRIE is a technique with which deep and narrow structures with vertical sidewalls can be etched in silicon. ${ }^{21}$ The chip has dimensions of $16 \times 20 \mathrm{~mm}^{2} \times 350 \mu \mathrm{m}$, as shown in Fig. 1. The channel system includes two inlets (I1 and I2), through which two different reactant gas mixtures can be introduced and mixed on-chip. In addition, two outlets (O1 and $\mathrm{O} 2)$ are included in 
the chip. The inlets and outlets all consist of $600 \mu \mathrm{m}$ diameter holes which extend from the front side to the back of the chip. The two inlets are connected to a mixing channel of depth $250 \mu \mathrm{m}$. To make certain that the two reactant gas mixtures are properly mixed when entering the reaction zone, the mixing channel has a long meander structure and a width of only $150 \mu \mathrm{m}$. The mixing channel connects to $\mathrm{O} 1$ through a channel with the same depth as the mixing channel. To achieve a high flow conductance, this channel has a width of $500 \mu \mathrm{m}$. The gas flow through the mixing channel enters the chip through I1 and I2 and leaves the microreactor through O1. This gas flow is typically on the order of $10 \mathrm{Nml} \mathrm{min}{ }^{-1}\left(4.1 \times 10^{18}\right.$ molecules s$\left.{ }^{-1}\right)$.

A channel leads from the mixing channel to a circular reaction chamber with a diameter of $1.0 \mathrm{~cm}$, and another channel leads from the reaction chamber to $\mathrm{O} 2$. These two channels as well as the reaction chamber all have depths of only $3 \mu \mathrm{m}$. In experiments, the catalyst is contained within the reaction chamber, and the chemical reaction occurs here. Gas flows from the mixing channel into the reaction chamber and proceeds to $\mathrm{O} 2$. The low depth yields a reaction chamber volume of only $240 \mathrm{nl}$. The purpose of the shallow channel connecting the mixing channel to the reaction chamber is to limit back-diffusion of reaction products to the mixing channel. O2 is connected to a QMS, which analyzes the composition of the gas leaving the reaction chamber. Thus, the entire gas flow through the reaction chamber, and consequently almost all reaction products, enter this QMS. Only reaction products which might back diffuse from the reaction chamber into the mixing channel can elude the QMS.

The QMS is a vacuum system from which gas can only be pumped at a limited pumping speed. For this reason, the connection between the reaction chamber and $\mathrm{O} 2$ includes an on-chip flow-limiting capillary with a low flow conductance. The capillary has a width of $5 \mu \mathrm{m}$, a depth of $3 \mu \mathrm{m}$, and a length of $1500 \mu \mathrm{m}$. To facilitate optimal detection, these dimensions of the capillary have been chosen to obtain a gas flow through the reaction chamber of around $3 \times 10^{14}$ molecules s $^{-1}$ and a resulting QMS pressure of around $10^{-7}$ mbar under typical experimental conditions.

In the experimental setup, the absolute pressure at $\mathrm{O} 1$ can be varied in the range of $0.1-5.0$ bar. The absolute pressure at $\mathrm{O} 2$ is at vacuum level since this outlet is connected to the QMS. It follows that there is a pressure difference of the order of 1 bar across the system. Since the flow conductance of the capillary is much lower than the conductance of any other part of the channel system, almost the entire pressure drop occurs across the capillary. Consequently, the pressure in the reaction chamber is expected to be very close to the controlled pressure at $\mathrm{O} 1$.

The gas flow through the reaction chamber is only around $0.01 \%$ of the flow through the mixing channel because the flow resistance of the capillary is much higher than that of the mixing channel. This difference in magnitudes of the gas flows is desirable for the following reasons. Since the gas flow through the reaction chamber constitutes the entire flow entering the QMS, this flow determines the pressure in the QMS. Only when the reaction chamber gas flow has an approximate magnitude of $10^{14}$ molecules s ${ }^{-1}$ will the QMS pressure be compatible with mass spectrometer operation. Concerning the mixing channel, such a low gas flow would be difficult to control with off-the-shelf mass flow controllers (MFCs) and highly impractical when changing the composition of the gas mixture introduced through the inlets. The volume of the exterior gas supply system connected to I1 and I2 in the experimental setup is much larger than the volume of the microchannel system itself. When changing the composition of the gas entering the chip, one, therefore, has to wait for the old gas to be fully washed away from the exterior tubing by the new gas. Until then, the composition of the gas entering the chip does not resemble the one specified by the user. Thus, a relatively high mixing channel gas flow is required to ensure a short gas residence time in the exterior tubing and a practical time-scale for changes of the gas mixtures. For these reasons, $\mathrm{O} 1$ has been included in the chip to generate a bypass gas flow on the order of $10^{18}$ molecules s ${ }^{-1}$. A gas flow of this magnitude can readily be controlled with off-the-shelf MFCs.

In summary, reactant gases are introduced into the reactor through the two inlets. The reactant gas mixture flows through the mixing channel to O1, where it is vented to the atmosphere. However, around $0.01 \%$ of the mixing channel gas flow proceeds to the reaction chamber, where the reactants can react and form products under influence of the catalyst. The resulting gas mixture flows from the reaction chamber through the capillary and enters the QMS, where the gas composition is analyzed.

The large area of the reaction chamber increases the amount of catalyst which can fit at the floor of the reaction chamber for a given density. This is favorable for the purpose of obtaining a reasonably high reaction rate when the turnover frequency is low or when a low catalyst density is required to avoid sintering. The circular shape is an advantage since it makes confinement of the catalyst deposition to the reaction chamber easier than it would be, if for instance a long, narrow meander geometry of the same large area were used. When depositing metal clusters from a magnetron sputtering cluster source, the large, circular shape makes it fairly easy to align a shadow mask. When depositing a catalyst manually with a pipette, the high area and circular shape also ease the process. In addition, with a circular design liquid catalyst suspensions deposited in the reactor form a relatively uniform loading. Finally, the circular shape allows easy use of the "locally cooled anodic bonding" method which has been developed for low-temperature sealing of the microreactor. ${ }^{22}$ This bonding method is very useful for catalysts, such as gold clusters, which are likely to sinter at elevated temperatures.

After deposition of catalyst in the catalyst bed, the reactor is sealed with a Pyrex lid, using anodic bonding. ${ }^{23}$ During the bonding process, the Pyrex-lid and the floor of the reaction chamber will be attracted toward each other by a strong electrostatic force. Because of the low depth-to-diameter ratio of the reaction chamber, the attractive force might cause the Pyrex-lid to get into intimate contact with the floor of the reaction chamber during the anodic bonding. If this happens, attractive surface forces will quickly cause the entire reaction chamber to collapse, permanently bonding the lid to the re- 
1

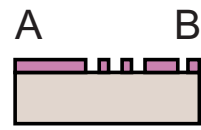

2

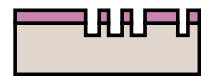

3
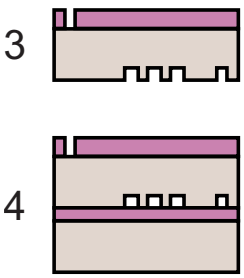

8

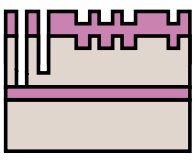

5

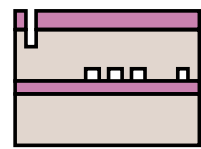

10
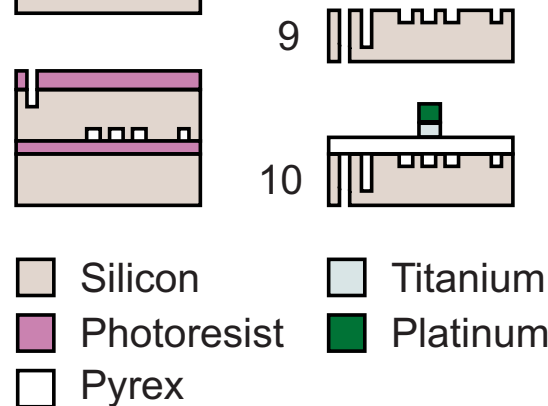

FIG. 2. (Color online) Fabrication sequence for the microreactor. (1) Reaction chamber, shallow channels, and capillary are defined in photo resist; (2) etching of the reaction chamber, shallow channels, and capillary; (3) inlet and outlet holes are defined in photoresist from back; (4) handle wafer mounting; (5) etching of inlet and outlet holes; (6) inlet and outlet holes and mixing channel are defined from front side; (7) handle wafer mounting; (8) etching of inlet and outlet holes and mixing channel; (9) removal of remaining photoresist and handle wafer; and (10) reactor sealed with Pyrex lid with integrated temperature detector using anodic bonding.

action chamber floor. ${ }^{24-26}$ To avoid such a collapse, the reaction chamber contains $200 \mu \mathrm{m}$ diameter silicon pillars which are spaced $1 \mathrm{~mm}$ apart. These pillars prevent the lid from getting into contact with the reaction chamber floor during the bonding process.

\section{B. Microreactor fabrication}

The microreactor is fabricated using micromanufacturing batch techniques. With these processes, large numbers of reactors can be produced in a short time and at relatively low cost. The silicon chips are fabricated using ultraviolet (UV) lithography, RIE, and DRIE. These are all standard microfabrication processes. The fabrication sequence for the silicon chips is shown in Fig. 2. The starting point is a $350 \mu \mathrm{m}$ thick silicon wafer. First, what will later become the substrate front side is covered with a $1.5 \mu \mathrm{m}$ thick layer of AZ5214 photoresist (a photosensitive, etch resistant polymer). A pattern containing the reaction chamber and the capillary is then defined in the polymer using UV lithography, and the wafer is etched to a depth of around $3.0 \mu \mathrm{m}$ through a RIE process. This pattern also includes the two microchannels leading from the mixing channel to the reaction chamber and from the reaction chamber to the capillary, respectively. After etching the low flow part of the channel system, the polymer mask is removed in acetone. A $9.5 \mu \mathrm{m}$ thick layer of AZ4562 photoresist is then applied to the back of the

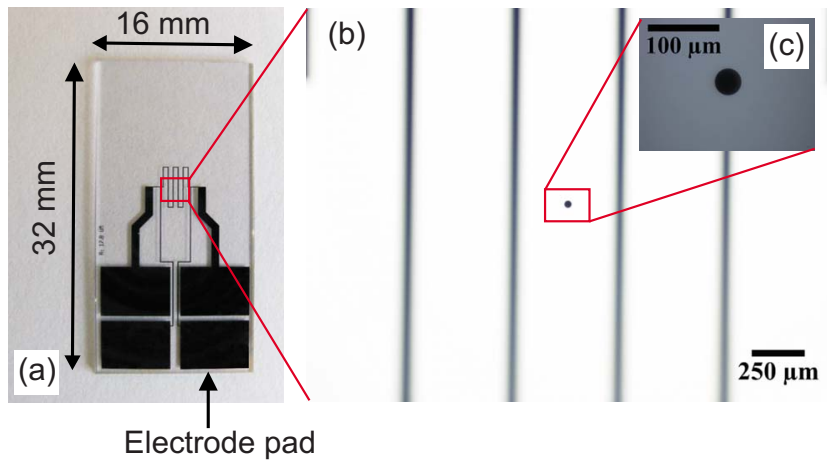

FIG. 3. (Color online) (a) Photograph of the Pyrex lid with integrated platinum thin film RTD. The temperature is determined with a four-point-probe measurement of the resistance of the meander path in the center of the lid. (b) Magnification of the center of the meander path. The platinum catalyst circle is visible in the center. (c) Magnification of the catalyst circle. This particular circle has a diameter of $36 \mu \mathrm{m}$.

wafer, and a pattern containing the in- and outlet holes is defined using UV lithography. Now, the front of the wafer is bonded to a handle wafer using photoresist as an adhesive. This is done to protect this side of the wafer, which might otherwise become damaged along the edge during the subsequent DRIE process. The pattern is then etched using DRIE to a depth of around $100 \mu \mathrm{m}$, followed by removal of the remaining photoresist and the handle wafer in acetone. The front of the wafer is now covered with a $9.5 \mu \mathrm{m}$ thick layer of AZ4562 photoresist, and a pattern containing the mixing channel is defined using UV lithography. Again, before the subsequent DRIE process, the side of the wafer not covered by photoresist is bonded to a handle wafer. At this point in the process flow, the handle wafer is required since the inand outlet holes will now be etched all the way through the wafer. In the DRIE tool helium gas is used to cool the back of the wafer during etching. If no handle wafer were used, the helium gas would leak into the plasma chamber through the in- and outlet holes. The mixing channel pattern is now etched using DRIE until it reaches the in- and outlet holes at a depth of approximately $250 \mu \mathrm{m}$. Afterwards, the remaining photoresist and the handle wafer are removed in acetone. As a final fabrication step, the chip is thermally oxidized with an oxide thickness of $50 \mathrm{~nm}$. The wafer is sawn into small chips, each containing one microreactor.

To prepare the microreactor for a catalyst test experiment, a catalyst material can be deposited in the reaction chamber using one of the deposition methods described earlier. The microchannel system is subsequently sealed with a Pyrex lid using anodic bonding. This concludes the preparation procedure, and catalyst testing can be initiated. The total preparation time before measurements can be commenced depends on the catalyst deposition method, but is usually around one hour. In the experimental results presented in this article, the catalyst is incorporated on the Pyrex lid surface as a thin film. Thus, no additional catalyst is deposited in the reaction chamber prior to bonding in these specific experiments.

The side of the Pyrex-lid facing the silicon chip includes a circular platinum thin film, as seen in Fig. 3. This platinum circle acts as a catalyst in the test reaction. A number of 
1
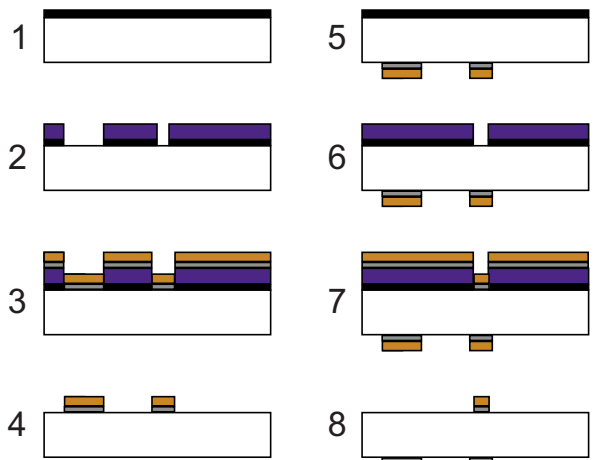

Pyrex

Aluminum $\square$ Photoresist
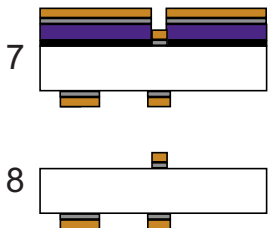

Titanium

Platinum

FIG. 4. (Color online) Fabrication sequence for the Pyrex lid. (1) Aluminum thin film deposition on front side; (2) RTD is defined in photoresist and aluminum; (3) titanium and platinum thin film deposition; (4) lift-off of titanium and platinum and removal of aluminum; (5) aluminum thin film deposition on back; (6) catalyst circle is defined in photoresist and aluminum; (7) titanium and platinum thin film deposition; and (8) lift-off of titanium and platinum and removal of aluminum.

different lids with platinum circle areas ranging from $15 \mu \mathrm{m}^{2}$ to $5000 \mu \mathrm{m}^{2}$ is fabricated. By performing measurements with different platinum circle areas, it is possible to investigate the relation between the catalyst area and the lowest temperature at which catalytic activity can be detected. The other side of the lid is equipped with an integrated four-point probe resistive temperature detector (RTD) consisting of a structured platinum thin film. The positions of the RTD and the catalyst circle both match with the center of the reaction chamber. The Pyrex lid has dimensions of $16 \times 32 \mathrm{~mm}^{2} \times 500 \mu \mathrm{m}$.

The lid is fabricated using UV lithography, PVD, and lift-off processes in acetone. These are all standard microfabrication processes. The fabrication sequence for the lid is shown in Fig. 4. The starting point is a $500 \mu \mathrm{m}$ thick Pyrex wafer. First, what will later become the RTD side of the lid is covered with a $10 \mathrm{~nm}$ thick aluminum thin film using e-beam PVD. The aluminum layer acts to promote adhesion between photoresist and the wafer. In addition, the aluminum layer reflects UV light and thus prevents reflections from the chuck in the exposure tool during the lithography process. This is important since the Pyrex wafer is transparent to UV light. A $1.5 \mu \mathrm{m}$ thick layer of AZ5214 photoresist is now spun on top of the aluminum, and a pattern containing the RTDs is subsequently defined in the polymer using UV lithography. As a part of the lithography-process the photoresist is developed in an aqueous $\mathrm{NaOH}$-solution. During this development process, the aluminum below the exposed parts of the photoresist is also etched away. A $10 \mathrm{~nm}$ thick titanium thin film is then deposited on the RTD side using e-beam PVD. This titanium layer serves as an adhesion layer between the Pyrex substrate and the platinum thin film which is deposited next. Now, a $100 \mathrm{~nm}$ thick platinum thin film is deposited using e-beam PVD. The polymer mask and the metal on top of it are subsequently lifted off in acetone using ultrasound. This is followed by removal of the remaining

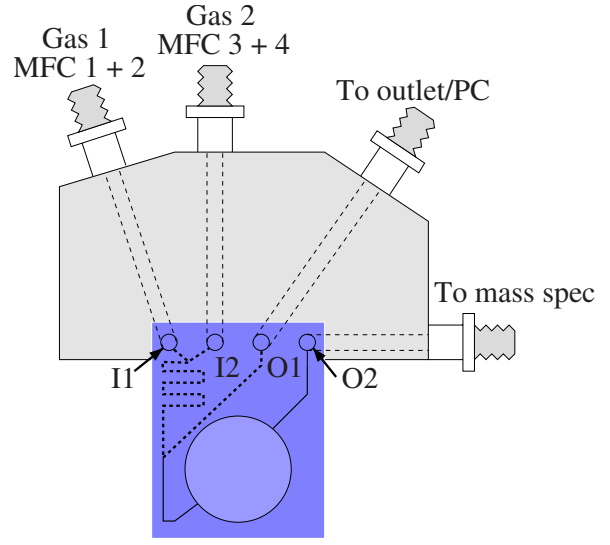

FIG. 5. (Color online) Sketch of the stainless steel manifold fixture for the microreactor. Gas lines machined in the interior of the fixture connect the inlets and outlets of the microreactor to MFCs, a PC, and a QMS.

aluminum in an aqueous solution of $\mathrm{NaOH}$. The RTD has now been formed on top of the Pyrex lid. Afterwards, the catalyst circle is formed on the opposite side of the lid. This is done by processing the back of the wafer in exactly the same way as the front side, while using a different mask when exposing the polymer to UV light. Hence, the catalyst circle consists of a $100 \mathrm{~nm}$ thick platinum thin film in addition to a $10 \mathrm{~nm}$ thick titanium thin film in between the platinum and the Pyrex substrate. Finally, the Pyrex wafer is sawn into small chips, each containing one lid. Before the measurements, the RTDs are annealed at $400{ }^{\circ} \mathrm{C}$.

\section{Experimental setup}

The gas handling system is made from 1/4 in. stainless steel tubing with welded $\mathrm{VCR}^{\circledR}$ (Swagelok) fittings as connections. The microreactor is mounted in a stainless steel manifold fixture with welded $\mathrm{VCR}^{\circledR}$ fittings, and the gas lines are connected to the microreactor through this fixture. A sketch of the arrangement is shown in Fig. 5. The gas lines are machined in the interior of the fixture, and tight connections between the steel block and the microreactor are ensured by using Kalrez perfluoroelastomer O-rings.

Since the O-rings are made from an elastomer, a small amount of gas diffusion from the surrounding air into the system is unavoidable, particularly at elevated temperatures. This gives rise to unwanted background signals of $\mathrm{O}_{2}$ and $\mathrm{N}_{2}$, complicating analysis of the reaction products and source gas species. To mitigate this problem, a continuous flow of argon is introduced in the volume surrounding the O-rings. Thus, any unwanted background due to diffusion through O-rings will be in the form of argon.

The reactor is heated from the silicon side using an external resistive heating element covering the outline of the reaction chamber. The temperature is measured with four point measurements of the resistance of the integrated RTD. The gas flow to the two inlets is controlled by four MFCs allowing mixing of up to four different gases. Outlet 1 is connected to a pump via a pressure controller (PC). This makes experiments at absolute pressures from 0.1 to 5.0 bar possible. The gas flows, the PC, and the temperature are all controlled with a LabVIEW program. The temperature is 
controlled using a PID algorithm, allowing temperature changes of up to $1 \mathrm{~K} / \mathrm{s}$ and constant temperatures within $0.1 \mathrm{~K}$. Time resolved gas detection is performed with a QMS (Balzers QMA 125) equipped with a secondary electron multiplier.

\section{RESULTS AND DISCUSSION}

\section{A. Gas flow}

The pressure difference between the reaction chamber and $\mathrm{O} 2$ gives rise to a gas flow through the capillary, and the magnitude of the gas flow depends on the exact value of the reaction chamber pressure as well as the temperature. The absolute gas flow through the capillary is an essential design parameter for the following reasons. The reaction chamber and the capillary are connected in series, so the flows through them are the same. Consequently, the residence time of the gas in the reaction chamber can be derived from the capillary flow. Furthermore, knowledge of the gas flow is important for interpretation of experimental results, for instance for determining absolute reaction rates. Finally, when designing the microreactor, the capillary dimensions must be chosen carefully to ensure that the flow to the QMS facilitates optimal gas composition analysis. Gas flows through narrow capillaries with vacuum pressure at one end are nontrivial since the small cross sectional dimensions might be comparable to the mean free path of the gas, resulting in a nonviscous flow regime. It follows that it is of interest to measure the capillary gas flow using an absolute method.

To characterize the relation between the pressure in the reaction chamber and the gas flow through the capillary, a microreactor is mounted in the manifold fixture to allow flow from a fixed volume to the QMS. This is done by sealing up the gas lines in the interior of the fixture from the external pump system. Thus, a fixed volume constituted by the microchannel system in the reactor and the gas lines in the interior of the fixture is hermetically sealed. The only path by which gas can leave the volume is by entering the QMS through the on-chip capillary. In this experiment, a Baratron (MKS Inc. type 211, 0-1 bar range), which measures the pressure inside the fixed volume, is installed in the fixture. At the onset of the experiment, the fixed volume contains atmospheric air at atmospheric pressure, and the QMS is at vacuum pressure. The fixed volume is $11.2 \mathrm{ml}$, and the experiment is carried out at room temperature. The pressure is plotted as a function of time in Fig. 6(a). As gas flows through the capillary, the pressure in the fixed volume decreases, and the flow is obtained from

$$
\dot{N}(t)=\frac{V}{k_{B} T} \frac{d p_{v}(t)}{d t},
$$

where $\dot{N}(t)$ is the net number of gas molecules crossing a cross section of the capillary per unit time, $p_{v}(t)$ is the pressure in the fixed volume, $V$ is the volume, $k_{B}$ is the Boltzmann constant, $T$ is the temperature of the fixed volume, and $t$ is time. In the experiment the microreactor has a capillary depth of $3.0 \mu \mathrm{m}$. In Fig. $6(\mathrm{~b}), \dot{N}$ is plotted as a function of the pressure in the fixed volume.
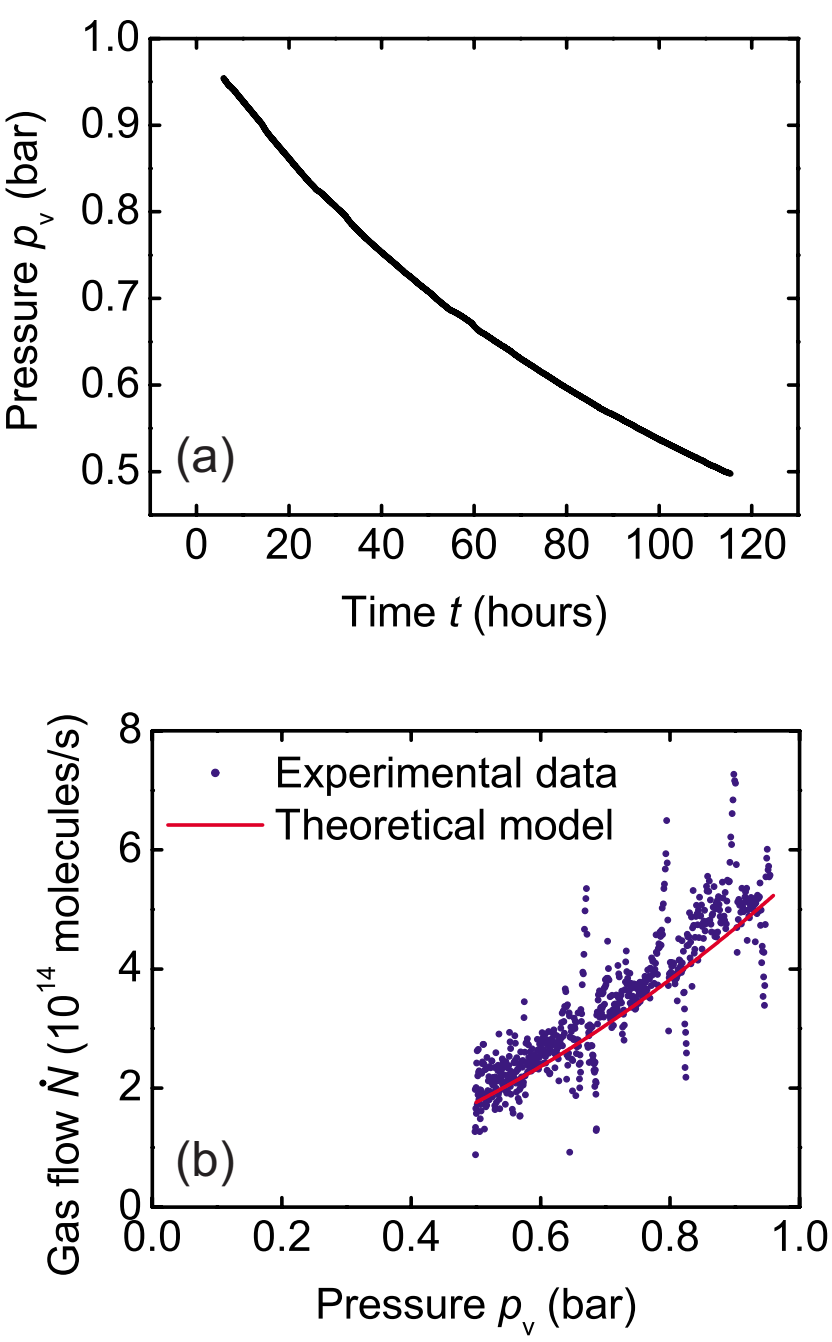

FIG. 6. (Color online) (a) Measured pressure $p_{v}$ in the fixed volume as a function of time. (b) Experimentally measured flow as a function of the reaction chamber pressure $p_{v}$ (blue circles). The red curve is the theoretically predicted flow as a function of $p_{v}$.

The gas flow through the capillary is now described with a theoretical model for comparison with the experimental results. The width of the capillary is very small compared to the widths of the channel leading from the mixing channel to the reaction chamber and the channel leading from the reaction chamber to the capillary. Consequently, the flow resistances of the latter two channels as well as that of the reaction chamber can be safely neglected when predicting the flow entering the QMS. Thus, in this discussion only the flow resistance of the capillary is considered.

Gas flow in a tube of characteristic cross sectional width $d$ can be characterized by the Knudsen number $\lambda / d$, where $\lambda$ is the mean free path of molecules in the gas. If $\lambda / d<0.0091$, the flow is viscous, if $0.0091<\lambda / d<1$, the flow is intermediate, and if $\lambda / d>1$, the flow is molecular. ${ }^{27,28}$ The capillary has a rectangular cross section with dimensions $5 \times 3 \mu \mathrm{m}^{2}$. However, when attempting to predict the relation between the capillary gas flow and the pressure in the reaction chamber, it is convenient to treat the capillary as having a circular cross section. The advantage of this approximation is that for a circular cross section analytic expressions for the flow exist for all three flow regimes. In 


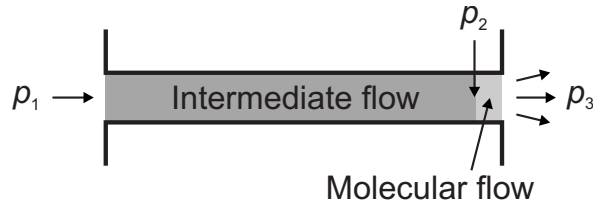

FIG. 7. Sketch of the capillary showing the reaction chamber side (left) and the QMS side (right). The reaction chamber pressure is $p_{1}$, and the QMS pressure is $p_{3}$. The regions with intermediate and molecular flow are indicated. At the position where the pressure is $p_{2}$, the gas flow changes from intermediate to molecular.

this discussion, a circular cross section of diameter $d=4.4 \mu \mathrm{m}$ and thus of the same area as the actual rectangular cross section will be assumed. by $^{27}$

For air at room temperature, the mean free path is given

$$
\lambda=\frac{6.7 \times 10^{-3} \mathrm{~Pa} \mathrm{~m}}{p},
$$

where $p$ is the pressure. For a capillary diameter of $4.4 \mu \mathrm{m}$, this means that the flow is intermediate at atmospheric pressure, and that the transition from intermediate to molecular flow occurs at a pressure of around $1.5 \times 10^{3} \mathrm{~Pa}$. In Fig. 7, the capillary is shown schematically with the two flow regimes. The inlet pressure is $p_{1}$, and at the pressure $p_{2}$ the flow changes from intermediate to molecular. The pressure in the QMS is denoted $p_{3}$.

In the intermediate flow regime, the gas flow through a tube of length $\ell$ with circular cross section is ${ }^{27}$

$$
\dot{N}=c \frac{p_{1}-p_{2}}{k_{B} T},
$$

with

$$
c=\frac{\pi}{128} \frac{\bar{p} d^{4}}{\eta \ell}+\frac{1}{3} \sqrt{\frac{\pi}{2} \frac{d^{3}}{\ell v}} \frac{1+\frac{d v \bar{p}}{\eta}}{1+1.24 \frac{d v \bar{p}}{\eta}},
$$

where $\eta$ is the viscosity of the gas, $\bar{p}=\left(p_{1}+p_{2}\right) / 2$, $v=\sqrt{m /\left(k_{B} T\right)}$, and $m$ is the mass of a single gas molecule. In the molecular flow regime ${ }^{29}$

$$
\dot{N}=\frac{1}{3} \sqrt{\frac{\pi}{2}} \frac{d^{3}}{\ell v} \frac{p_{2}-p_{3}}{k_{B} T} .
$$

Using Eq. (2), the expressions Eqs. (3) and (5) can be combined to obtain an analytical expression for the flow that includes both the intermediate and the molecular flow regimes. In addition, an analytical expression for the fraction of the capillary length where the flow is intermediate can be obtained. For atmospheric air at $T=295 \mathrm{~K}$ and at $p_{v}>0.5 \mathrm{bar}$, the flow is intermediate in more than $98 \%$ of the length of the capillary, according to this calculation. In Fig. 6(b), the theoretically predicted gas flow as a function of the pressure in the fixed volume is plotted with $T=295 \mathrm{~K}$, $\eta=17.8 \times 10^{-6} \mathrm{~Pa} \mathrm{~s}$, and $m=4.81 \times 10^{-26} \mathrm{~kg}$ (the average molecular mass in the air). Both the intermediate and the molecular flow regimes are taken into account when calculating this gas flow. A good agreement with the experimental data is observed. It is emphasized that the expected gas flow plotted in Fig. 6(b) is generated from a purely theoretical model, and that the experimental data are not considered when making this prediction. Thus, the theoretical model presented here is not a fit to experimental data but rather a prediction based entirely on the capillary dimensions, the temperature, and the properties of the gas.

Based on these results, the capillary gas flow at typical experimental conditions can be inferred. It appears from Fig. 6(b) that, at approximately atmospheric pressure in the reaction chamber, the capillary gas flow is around $6 \times 10^{14}$ molecules s ${ }^{-1}$ at room temperature. At the same pressure and a typical reactor temperature of $250{ }^{\circ} \mathrm{C}$, the theoretical model predicts a flow of around $3 \times 10^{14}$ molecules s ${ }^{-1}$, corresponding to a gas residence time in the reaction chamber of around $11 \mathrm{~s}$.

During operation, gas is pumped out of the QMS by a turbo pump. In steady state, the amount of gas flowing through the capillary equals the amount pumped by the turbo pump. The pressure $p_{\mathrm{ms}}$ in the QMS is then given by

$$
p_{\mathrm{ms}}=\frac{k_{B} T_{\mathrm{ms}}}{S} \dot{N},
$$

where $T_{\mathrm{ms}}$ is the temperature of the gas in the QMS, $\dot{N}$ is the number of gas molecules pumped by the turbo pump per unit time, and $S$ is the gas volume pumped by the turbo pump per unit time. $S$ is referred to as the pumping speed. It is clear from Eq. (6) that the gas flow through the capillary determines the pressure in the QMS. Since the gas flow in turn is determined by the capillary dimensions, these dimensions must be chosen to facilitate a suitable QMS pressure. During the experiment, $p_{\mathrm{ms}}$ is continuously measured with an ion gauge and is in the range $2-10 \times 10^{-7}$ mbar. These pressures are well-suited for QMS analysis suggesting that the capillary dimensions are appropriate. Using Eq. (6), the effective pumping speed is determined to be approximately $38 \mathrm{l} / \mathrm{s}$. This is reasonable, since the geometric pump aperture is about $9 \mathrm{~cm}^{2}$ giving a maximum theoretical pumping speed of $\sim 106 \mathrm{l} / \mathrm{s}$.

Although the experimental data in Fig. 6(b) are in good agreement with the theoretical prediction, pronounced deviations appear at $p_{v}=67 \mathrm{kPa}, p_{v}=80 \mathrm{kPa}$, and $p_{v}=90 \mathrm{kPa}$. These deviations derive from the numerical differentiation of the pressure with respect to time in Eq. (1). The deviations are caused by distinct periods of time in the originally measured values of $p_{v}(t)$ characterized by a relatively abrupt increase of about $200 \mathrm{~Pa}$ in $p_{v}$ followed by a corresponding decrease back to the original level 5-8 $\mathrm{h}$ later. These "bumps" in the measured values of $p_{v}(t)$ are caused by unstable performance of the Baratron. In spite of this effect, the quantitative agreement between experimental data and the theoretical prediction clearly suggests that the gas flow can be understood in terms of the theoretical model outlined above.

It is noted that other desired values of the capillary flow resistance can easily be obtained by fabricating microreactors with other capillary dimensions. This could be favorable for some experiments. For a constant inlet pressure, an in- 


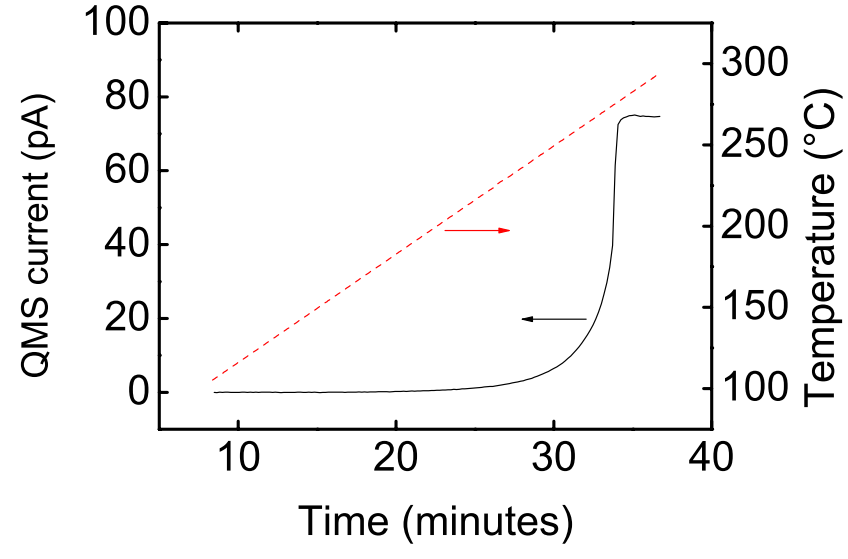

FIG. 8. (Color online) Experimental data from oxidation of carbon monoxide in the microreactor using a platinum thin film catalyst. The measured temperature (red, dashed curve, right ordinate axis) and the $\mathrm{CO}_{2}$ signal in the QMS (black, solid curve, left ordinate axis) are plotted as a function of time. The catalyst area is $515 \mu \mathrm{m}^{2}$, and the $\mathrm{CO}_{2}$ content is measured using the mass 44 signal in the QMS.

crease of the capillary width would for example result in a shorter reaction chamber residence time.

\section{B. Oxidation of carbon monoxide}

To demonstrate the operation of the microreactor, $\mathrm{CO}$ oxidation on platinum thin film circles is used as a test reaction. In particular, the experiments are performed to test the ability to measure catalytic activity of catalysts with low surface areas. Since the main purpose of the reactor design is to improve sensitivity to enable studies on low density mass selected clusters, this is essential information on reactor performance. A gas mixture consisting of $\mathrm{CO}$ and $\mathrm{O}_{2}$ is used, and the platinum circles have different areas ranging from 15 to $5000 \mu \mathrm{m}^{2}$. The reaction is run at a pressure of $1.00 \mathrm{bar}$ in a surplus of oxygen, with an $\mathrm{O}_{2}$ : $\mathrm{CO}$ ratio of 20:1 and with a total flow rate of $8.4 \mathrm{Nml} \mathrm{min}^{-1}$ through the mixing channel. After mounting in the manifold fixture, the reactor is heated to $400{ }^{\circ} \mathrm{C}$ in the reaction gas, until any organic contaminants have been burned away, and the gas system has stabilized. The experiments consist of repeatedly ramping the temperature from 80 to $350{ }^{\circ} \mathrm{C}$ at a rate of $400 \mathrm{~K} / \mathrm{h}$, activating the $\mathrm{CO}$ oxidation

$$
2 \mathrm{CO}(g)+\mathrm{O}_{2}(g) \rightarrow 2 \mathrm{CO}_{2}(g) .
$$

Figure 8 shows the temperature and the $\mathrm{CO}_{2}$ signal in the QMS, both as a function of time, during a single temperature ramp. This experiment is carried out with a catalyst area of $515 \mu^{2}$, and the $\mathrm{CO}_{2}$ content in the QMS is measured using the mass 44 signal. As the temperature is increased, the $\mathrm{CO}_{2}$ signal rises exponentially until a "light off" phenomenon is seen, and full conversion of $\mathrm{CO}$ to $\mathrm{CO}_{2}$ takes place. In Fig. 8, the background of $\mathrm{CO}_{2}$ from reactions on the filament in the QMS has been subtracted. The $\mathrm{CO}$ and $\mathrm{O}_{2}$ signals are measured simultaneously with the $\mathrm{CO}_{2}$ signals at mass 28 and 32, respectively. Both signals drop as the $\mathrm{CO}_{2}$ content increases in accordance with the stoichiometry of the reaction.

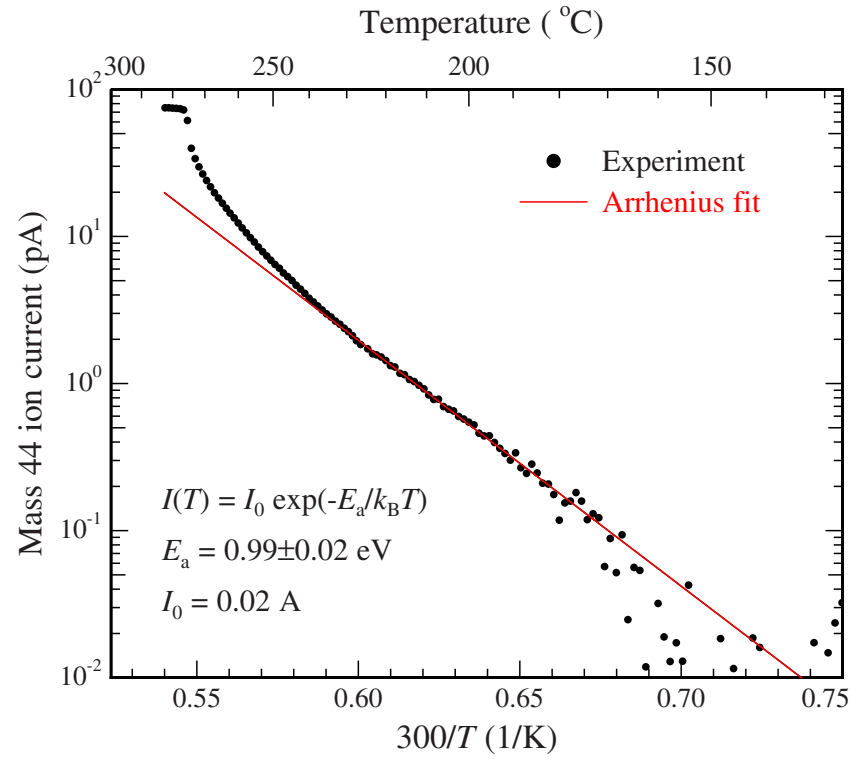

FIG. 9. (Color online) Arrhenius plot of the mass $44\left(\mathrm{CO}_{2}\right)$ signal plotted in Fig. 8 (black circles). The catalyst area for this experiment is $515 \mu \mathrm{m}^{2}$ platinum. A linear fit of the part of the experimental data, where the reaction exhibits Arrhenius behavior, is also shown (red line). The slope of the fit corresponds to an apparent activation energy of $0.99 \mathrm{eV}$.

At low conversion, the $\mathrm{CO}_{2}$ reaction rate $\dot{N}_{\mathrm{CO}_{2}}$ (the number of $\mathrm{CO}_{2}$ molecules produced in the reaction chamber per unit time) is ${ }^{1}$

$$
\dot{N}_{\mathrm{CO}_{2}} \simeq A r_{0}\left(p_{\mathrm{CO}}, p_{\mathrm{O}_{2}}, p_{\mathrm{CO}_{2}}\right) \exp \left(\frac{-E_{a}}{k_{B} T}\right),
$$

where $A$ is the area of the platinum circle, $E_{a}$ is the apparent activation energy, and $p_{\mathrm{CO}}, p_{\mathrm{O}_{2}}$, and $p_{\mathrm{CO}_{2}}$ are the partial pressures in the reaction chamber of $\mathrm{CO}, \mathrm{O}_{2}$, and $\mathrm{CO}_{2}$, respectively. $r_{0}\left(p_{\mathrm{CO}}, p_{\mathrm{O}_{2}}, p_{\mathrm{CO}_{2}}\right)$ is a parameter which depends on the partial pressures and the catalyst. The data from Fig. 8 are shown as an Arrhenius plot in Fig. 9. Here, it can be seen that at low temperature and low conversion the rate follows an Arrhenius behavior as expected, with an apparent activation energy $E_{a}$ of $0.99 \mathrm{eV}$. For all the samples, the reactivity exhibited Arrhenius behavior with apparent activation energies in the range $0.91-1.40 \mathrm{eV}$ with the highest values found for the measurements with smaller catalyst areas. Contreras et al. ${ }^{30}$ performed experiments with $\mathrm{CO}$ oxidation on a $15 \mathrm{~nm}$ thick platinum thin film deposited using PVD on a silicon(100) wafer with native oxide. At temperatures below "light off," these authors measured an apparent activation energy of $1.17 \mathrm{eV}$, within range of the values found in this work.

The microreactor and the corresponding experimental arrangement are designed to facilitate high sensitivity characterization of catalytic reactions. The sensitivity of the setup is now evaluated by considering the smallest catalyst area for which catalytic activity can be detected. The criterion for detection of catalytic activity is that the product QMS current $I_{p}$ must be distinguishable from the background in the QMS. Thus, $I_{p}$ must be higher than some threshold value $I_{t}$, which is determined by the QMS background. For the $\mathrm{CO}$ oxidation measurements described here, $I_{t}$ is defined to be $1.0 \mathrm{pA}$, 


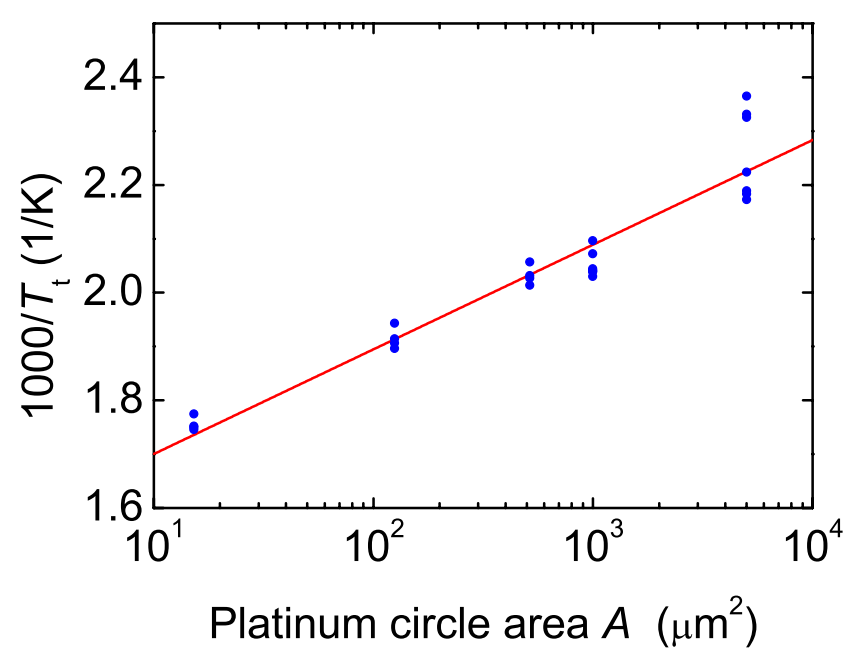

FIG. 10. (Color online) The inverse of the lowest temperature at which CO oxidation can be detected plotted as a function of catalyst area. Experimental data from all measured samples are shown (blue circles). The abscissa axis has logarithmic scale. The linear fit (red line) yields an apparent activation energy of $1.02 \mathrm{eV}$.

since this is significantly higher than the background. The product current increases linearly with the reaction rate $\dot{N}_{p}$ in the reaction chamber. It follows that the criterion for detection is satisfied when

$$
\dot{N}_{p}>\dot{N}_{t}
$$

where $\dot{N}_{t}$ is the reaction rate which causes a product current $I_{t}$.

From Eqs. (8) and (9), it follows that for a given area the temperature must exceed a certain threshold temperature $T_{t}$ before product formation can be detected. $T_{t}$ is the temperature which causes a reaction rate $\dot{N}_{t}$. Only if the temperature is higher than $T_{t}$, will the catalytic activity be high enough to cause a QMS product signal distinguishable from the background. In view of Eqs. (8) and (9), the threshold temperature can be predicted.

$$
\dot{N}_{t}=A r_{0} \exp \left(\frac{-E_{a}}{k_{B} T_{t}}\right) \Rightarrow \frac{1}{T_{t}}=\frac{k_{B}}{E_{a}}\left(\ln A-\ln \frac{\dot{N}_{t}}{r_{0}}\right) .
$$

Assuming that $\dot{N}_{t} / r_{0}$ may be considered constant, only $A$ and $T_{t}$ vary in the $\mathrm{CO}$ oxidation experiments. Thus, the inverse threshold temperature plotted against the logarithm of the area should yield a straight line with a slope of $k_{B} / E_{a}$. Figure 10 shows the experimental data from $\mathrm{CO}$ oxidation in the microreactors. The inverse of the lowest temperature at which $\mathrm{CO}_{2}$ formation can be detected is plotted against the platinum circle area (logarithmic scale). In the data evaluation, the threshold current $I_{t}$ is defined to be $1.0 \mathrm{pA}$, which is significantly above the background (see Fig. 9). From the slope of the best linear fit to the data, an apparent activation energy of $1.02 \mathrm{eV}$ is found. This is within range of the values found from the individual Arrhenius plots.

It appears from Fig. 10 that even for the smallest catalyst area of $15 \mu \mathrm{m}^{2}$, conversion into $\mathrm{CO}_{2}$ can be detected at a temperature of $\sim 300{ }^{\circ} \mathrm{C}$. This temperature can easily be reached with the experimental setup. An area of $15 \mu \mathrm{m}^{2}$ is considerably lower than catalyst areas for other high- sensitivity chemical reactors reported in literature. ${ }^{9,10}$ The experimental data thus demonstrate the high sensitivity of the microreactor and the capability to measure reactivity of small amounts of catalyst.

\section{CONCLUSIONS}

The microfabricated chemical reactor presented here facilitates highly sensitive catalyst testing. An integrated capillary limits the gas flow through the catalyst bed to around $3 \times 10^{14}$ molecules s $^{-1}$ at typical experimental conditions, making it possible to direct the entire reaction zone gas flow to enter a QMS. This prevents unnecessary dilution of the reaction products and causes almost all the products to enter the gas analysis tool, thus ensuring a high sensitivity. The microreactor can be employed as a platform for testing of a wide variety of different catalysts, including industrial catalysts. However, the primary aim of the device is to allow measurements of catalytic activity and surface kinetics of model catalysts, which can only be obtained in small quantities, thus necessitating high sensitivity to low surface areas. In particular, the reactor is well-suited for characterization of mass selected metal clusters from a sputtering cluster source.

The relationship between the reaction zone gas flow and the pressure in the reaction chamber has been investigated with a theoretical model, which predicts the gas flow in the capillary to be in the intermediate flow regime. The theoretical model is in good agreement with experimental results. $\mathrm{CO}$ oxidation on low-area platinum thin films was carried out in the reactor as a test reaction to demonstrate the operation of the device. The experimental data show that for this reaction and under the conditions used in these experiments, catalysts with surface areas as low as $15 \mu \mathrm{m}^{2}$ can be conveniently characterized with the microreactor. This catalyst area is considerably lower than for other high-sensitivity chemical reactors reported in literature.

\section{ACKNOWLEDGMENTS}

Center for Individual Nanoparticle Functionality (CINF) is sponsored by The Danish National Research Foundation.

${ }^{1}$ I. Chorkendorff and J. Niemantsverdriet, Concepts of Modern Catalysis and Kinetics (Wiley, New York, 2003)

${ }^{2}$ K. F. Jensen, Chem. Eng. Sci. 56, 293 (2001).

${ }^{3}$ S. K. Ajmera, C. Delattre, M. A. Schmidt, and K. F. Jensen, Sens. Actuators B 82, 297 (2002).

${ }^{4}$ G. Kolb and V. Hessel, Chem. Eng. J. 98, 1 (2004).

${ }^{5}$ K. Jähnisch, V. Hessel, H. Lowe, and M. Baerns, Angew. Chem. Int. Ed. 43, 406 (2004).

${ }^{6}$ L. Kiwi-Minsker and A. Renken, Catal. Today 110, 2 (2005).

${ }^{7}$ K. Wong, S. Johansson, and B. Kasemo, Faraday Discuss. 105, 237 (1996).

${ }^{8}$ A. S. Eppler, G. Rupprechter, L. Guczi, and G. A. Somorjai, J. Phys. Chem. B 101, 9973 (1997).

${ }^{9}$ S. Johansson, E. Fridell, and B. Kasemo, J. Vac. Sci. Technol. A 18, 1514 (2000).

${ }^{10}$ P. W. Jacobs, S. J. Wind, F. H. Ribeiro, and G. A. Somorjai, Surf. Sci. 372, L249 (1997).

${ }^{11}$ U. Quaade, S. Jensen, and O. Hansen, Rev. Sci. Instrum. 75, 3345 (2004).

${ }^{12}$ O. Younes-Metzler, J. Svagin, S. Jensen, C. H. Christensen, O. Hansen, and U. Quaade, Appl. Catal., A 284, 5 (2005).

${ }^{13}$ O. Younes-Metzler, J. Johansen, S. Thorsteinsson, S. Jensen, O. Hansen, and U. Quaade, J. Catal. 241, 74 (2006).

${ }^{14}$ R. Z. Sørensen, A. Klerke, U. Quaade, S. Jensen, O. Hansen, and C. H. 
Christensen, Catal. Lett. 112, 77 (2006)

${ }^{15}$ U. Heiz and E. L. Bullock, J. Mater. Chem. 14, 564 (2004).

${ }^{16}$ K. Judai, S. Abbet, A. S. Worz, U. Heiz, and C. R. Henry, J. Am. Chem. Soc. 126, 2732 (2004)

${ }^{17}$ D. W. Goodman, Nature (London) 454, 948 (2008).

${ }^{18}$ S. Thybo, S. Jensen, J. Johansen, T. Johannessen, O. Hansen, and U. Quaade, J. Catal. 223, 271 (2004)

${ }^{19}$ S. Johansson, E. Fridell, and B. Kasemo, J. Catal. 200, 370 (2001).

${ }^{20}$ M. Roumanie, C. Delattre, F. Mittler, G. Marchand, V. Meille, C. de Bellefon, C. Pijolat, G. Tournier, and P. Pouteau, Chem. Eng. J. 135, S317 (2008).

${ }^{21}$ A. M. Hynes, H. Ashraf, J. K. Bhardwaj, J. Hopkins, I. Johnston, and J. N. Shepherd, Sens. Actuators, A 74, 13 (1999).
${ }^{22}$ P. C. K. Vesborg, J. L. Olsen, T. R. Henriksen, I. Chorkendorff, and O. Hansen, "Anodic bonding with cooling of heat sensitive areas," Rev. Sci. Instrum. (submitted)

${ }^{23}$ G. Wallis and D. I. Pomerantz, J. Appl. Phys. 40, 3946 (1969).

${ }^{24}$ W. P. Shih, C. Y. Hui, and N. C. Tien, J. Appl. Phys. 95, 2800 (2004).

${ }^{25}$ P. Mao and J. Y. Han, Lab Chip 5, 837 (2005).

${ }^{26}$ K. M. Knowles and A. T. J. van Helvoort, Int. Mater. Rev. 51, 273 (2006).

${ }^{27}$ A. Roth, Vacuum Technology, 3rd ed. (Elsevier, Amsterdam, 1990).

${ }^{28}$ U. Quaade, S. Jensen, and O. Hansen, J. Appl. Phys. 97, 044906 (2005).

${ }^{29}$ J. O'Hanlon, A User's Guide to Vacuum Technology, 3rd ed. (Wiley, Hoboken, New Jersey, 2003).

${ }^{30}$ A. M. Contreras, X. M. Yan, S. Kwon, J. Bokor, and G. A. Somorjai, Catal. Lett. 111, 5 (2006). 